\title{
EXP-FUNCTION METHOD FOR SOLVING NONLINEAR EVOLUTION EQUATIONS
}

\author{
Emine Misirli and Yusuf Gurefe \\ Department of Mathematics, Faculty of Science \\ Ege University, 35100 \\ Bornova, Izmir, Turkey \\ emine.misirli@gmail.com, ygurefe@gmail.com
}

\begin{abstract}
In this paper, we applied Exp-function method to some nonlinear evolution equations. The solution procedure of this method, by the help of symbolic computation of Matlab, Mathematica or so on, is of utter simplicity. The obtained results show that Exp-function method is very powerful and convenient mathematical tool for nonlinear evolution equations in science and engineering.
\end{abstract}

Key Words- Exp-function Method, Symbolic Computation, 2-D Bratu Type Equation, Generalized Fisher Equation

\section{INTRODUCTION}

The investigation of the wave solutions for nonlinear evolution equations plays an important role in the study of nonlinear physical phenomena. In recent years, many effective methods have been proposed for solving the nonlinear differential equations, such that tanh-sech method [1], extended tanh method [2], sine-cosine method [3], homogenous balance method [4], Jacobi elliptic function method [5], F-expansion method [6], homotopy perturbation method [7], variational iteration method [8], Hirotas bilinear methods [9], bifurcation method [10] and so on. In 2006, a new method, called Exp-function method, was first introduced by $\mathrm{He}$ and $\mathrm{Wu}$ [11], and was successfully studied in a lot of problems [12-20] and so on.

In this study, we apply Exp-function method to the two-dimensional Bratu-type equation given in [21] as

$$
u_{x x}+u_{y y}+\lambda \exp (s u)=0
$$

and the generalized Fisher equation with higher order nonlinearity given in $[22,23]$ as

$$
u_{t}=u_{x x}+u\left(1-u^{n}\right)
$$

Two-dimensional Bratu model stimulates a thermal reaction process in a rigid material, where the process depends on a balance between chemically generated heat addition and heat transfer by conduction [24]. The nonlinear reaction-diffussion equation was first introduced by Fisher as a model for the propagation of a mutant gene. It has wide application in the fields of logistic population growth, flame propagation, neurophysiology, autocatalytic chemical reactions, and nuclear reactor theory. It is well 
known that wave phenomena of plasma media and fluid dynamics are modelled by kink-shaped and tanh-solution or bell-shaped sech-solutions [25].

\section{EXP-FUNCTION METHOD AND APPLICATION TO THE TWO- DIMENSIONAL BRATU TYPE EQUATION}

Using a complex variation $\eta$ defined as $\eta=k x+w y$ and the transformation $u=(1 / s) \ln v$, we can convert Eq. (1) into ordinary different equation, which reads

$$
\left(k^{2}+w^{2}\right) v v^{\prime \prime}-\left(k^{2}+w^{2}\right)\left(v^{\prime}\right)^{2}+\lambda s v^{3}=0,
$$

where the prime denotes the derivative with respect to $\eta$.

We assume that the solution of Eq. (3) can be expressed in the form

$$
v(\eta)=\frac{\sum_{n=-c}^{d} a_{n} \exp (n \eta)}{\sum_{m=-p}^{q} b_{m} \exp (m \eta)},
$$

where $c, d, p$ and $q$ are positive integers, $a_{n}$ and $b_{m}$ are unknown constants.

For simplicity, we set $p=c=1$ and $q=d=1$, then Eq. (4) reduced to

$$
v(\eta)=\frac{a_{1} \exp (\eta)+a_{0}+a_{-1} \exp (-\eta)}{\exp (\eta)+b_{0}+b_{-1} \exp (-\eta)}
$$

Substituting Eq. (5) into Eq. (3), we have

$$
\frac{1}{A}\left[C_{4} \exp (4 \eta)+\cdots C_{0}+\cdots+C_{-4} \exp (-4 \eta)\right]=0 .
$$

Equating the coefficients of $\exp (n \eta)$ in Eq. (6) to be zero yields a set of algebraic equations

$$
\begin{aligned}
& C_{4}=0, \quad C_{3}=0, \quad C_{2}=0, \quad C_{1}=0, \quad C_{0}=0, \\
& C_{-1}=0, \quad C_{-2}=0, \quad C_{-3}=0, \quad C_{-4}=0 .
\end{aligned}
$$

Solving the system of algebraic equation given above, with the aid of symbolic computation system of Mathematica, we obtain

$$
a_{-1}=0, \quad a_{0}=a_{0}, \quad a_{1}=0, \quad b_{-1}=\frac{b_{0}^{2}}{4},
$$




$$
b_{0}=b_{0}, \quad w=\mp \sqrt{\left(\lambda n a_{0}-k^{2} b_{0}\right) / b_{0}} .
$$

Substituting Eq. (7) into Eq. (5), we obtain the following exact solution

$$
v(x, y)=\frac{a_{0}}{\exp (k x+w y)+b_{0}+\left(b_{0}^{2} / 4\right) \exp (-(k x+w y))} .
$$

So,

$$
u(x, y)=\ln \left[\frac{a_{0}}{\exp (k x+w y)+b_{0}+\left(b_{0}^{2} / 4\right) \exp (-(k x+w y))}\right]^{\frac{1}{s}} .
$$

Using the properties

$$
\exp (k x+w y)+\exp (-(k x+w y))=2 \cosh (k x+w y),
$$

when $b_{0}=\mp 2$, it is easy to see that Eq. (9) can reduce to travelling wave solution as follows:

$$
u(x, y)=\ln \left[\frac{a_{0}}{2 \cosh \left(k x \mp \sqrt{\left(\mp \lambda s a_{0}-2 k^{2}\right) / 2} y\right) \mp 2}\right]^{\frac{1}{s}} .
$$

\section{THE GENERALIZED FISHER EQUATION}

We now consider the generalized Fisher equation (2). Introducing the complex variation $\eta$ defined as $\eta=k x+w t$, we have

$$
k^{2} u^{\prime \prime}-w u^{\prime}-u^{n+1}+u=0
$$

where $k, w$ are real parameters and the prime denotes the derivative with respect to $\eta$. Making the transformation

$$
u=v^{\frac{1}{n}}
$$

Eq. (12) becomes

$$
k^{2} n v v^{\prime \prime}+k^{2}(1-n)\left(v^{\prime}\right)^{2}-w n v v^{\prime}-n^{2} v^{3}+n^{2} v^{2}=0 .
$$

We assume that the solution of Eq. (14) can be expressed in the form 


$$
v(\eta)=\frac{a_{1} \exp (\eta)+a_{0}+a_{-1} \exp (-\eta)}{\exp (\eta)+b_{0}+b_{-1} \exp (-\eta)} .
$$

Substituting Eq. (15) into Eq. (14), and by the help of Mathematica, equating to zero the coefficients of all powers of $\exp (n \eta),(n=-4,-3, \cdots, 3,4)$ yields a set of algebraic equations for $a_{-1}, a_{0}, a_{1}, b_{-1}, b_{0}, k, w$. Solving this system of algebraic equations by using Mathematica, we obtain the following results:

Case 1:

$$
\begin{aligned}
& a_{-1}=0, \quad a_{0}=0, \quad a_{1}=1, \quad b_{-1}=\frac{b_{0}^{2}}{4}, \quad b_{0}=b_{0}, \\
& k=\mp \frac{n}{\sqrt{2(n+2)}}, \quad w=-\frac{n(n+4)}{2(n+2)},
\end{aligned}
$$

where $b_{0}$ is a free parameter. Substituting these results into Eq. (15), we obtain the following exact solutions

$$
u(x, t)=\left[\frac{e^{k x+w t}}{e^{k x+w t}+b_{0}+\left(b_{0}^{2} / 4\right) e^{-k x-w t}}\right]^{\frac{1}{n}}
$$

Using the properties

$$
\begin{aligned}
& \exp (k x+w t)+\exp (-(k x+w t))=2 \cosh (k x+w t), \\
& \exp (k x+w t)-\exp (-(k x+w t))=2 \sinh (k x+w t),
\end{aligned}
$$

when $b_{0}=\mp 2, k$ and $w$, Eq. (17) becomes

$$
u(x, t)=\left[\frac{\cosh (\mp A(x \mp B t))+\sinh (\mp A(x \mp B t))}{\mp 2+2 \cosh (\mp A(x \mp B t))}\right]^{\frac{1}{n}},
$$

where $A=\frac{n}{\sqrt{2(n+2)}}$ and $B=\frac{n+4}{\sqrt{2(n+2)}}$. 


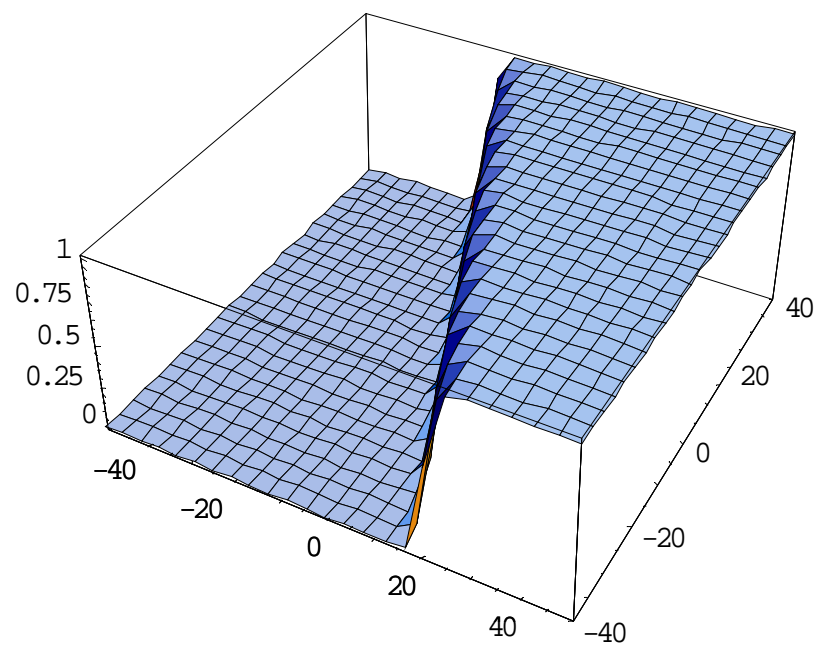

Figure 1 - Solution $u$ is shown at $b_{0}=2$ and $n=1$.

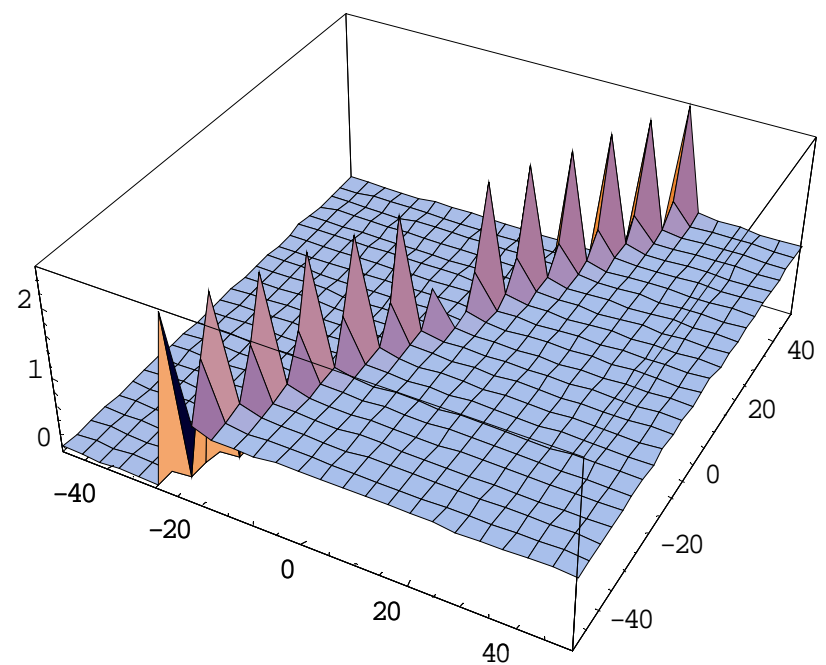

Figure 2 - Solution $u$ is shown at $b_{0}=-2$ and $n=1$.

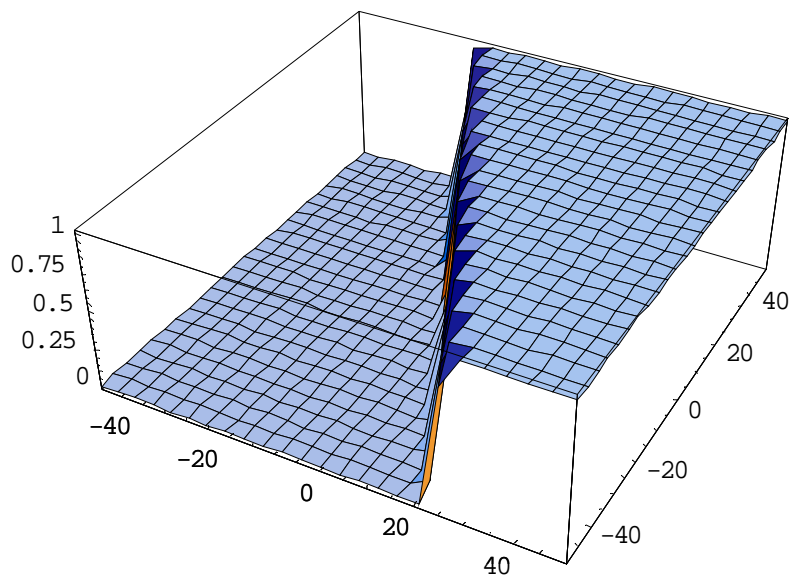

Figure 3 - Solution $u$ is shown at $b_{0}=2$ and $n=2$. 
Case 2:

$$
\begin{aligned}
& a_{-1}=b_{-1}, \quad a_{0}=0, \quad a_{1}=0, \quad b_{-1}=\frac{b_{0}^{2}}{4}, \quad b_{0}=b_{0}, \\
& k=\mp \frac{n}{\sqrt{2(n+2)}}, \quad w=-\frac{n(n+4)}{2(n+2)},
\end{aligned}
$$

where $b_{0}$ is a free parameter. Substituting these results into Eq. (15), we obtain the following exact solutions

$$
u(x, t)=\left[\frac{a_{-1} e^{-k x-w t}}{e^{k x+w t}+b_{0}+a_{-1} e^{-k x-w t}}\right]^{\frac{1}{n}}
$$

or

$$
u(x, t)=\left[\frac{\left(b_{0}^{2} / 4\right) e^{-k x-w t}}{e^{k x+w t}+b_{0}+\left(b_{0}^{2} / 4\right) e^{-k x-w t}}\right]^{\frac{1}{n}}
$$

Using the properties (18)-(19), when $b_{0}=\mp 2, k, w$, Eq. (23) becomes

$$
u(x, t)=\left[\frac{\cosh (\mp A(x \pm B t))-\sinh (\mp A(x \pm B t))}{\mp 2+2 \cosh (\mp A(x \pm B t))}\right]^{\frac{1}{n}},
$$

where $A=n / \sqrt{2(n+2)}$ and $B=(n+4) / \sqrt{2(n+2)}$.

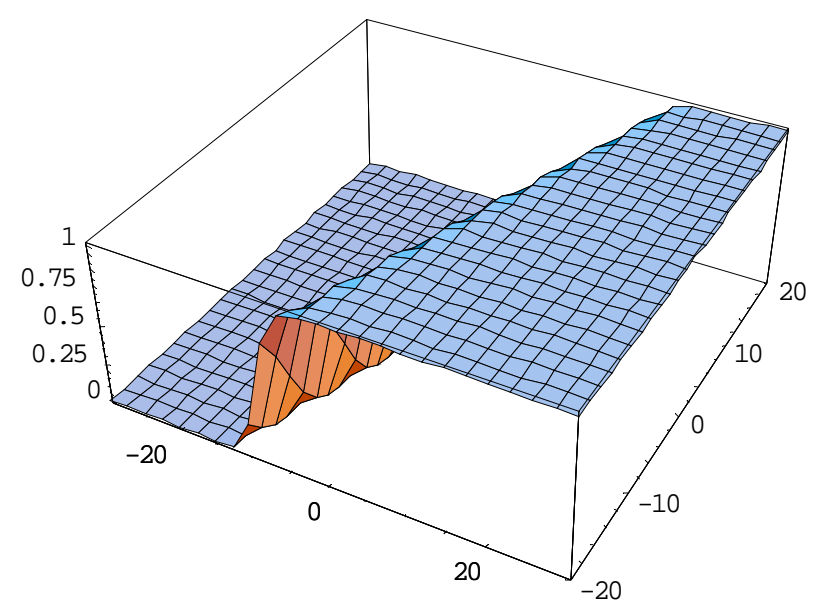

Figure 4 - Solution $u$ is shown at $b_{0}=2$ and $n=1$. 


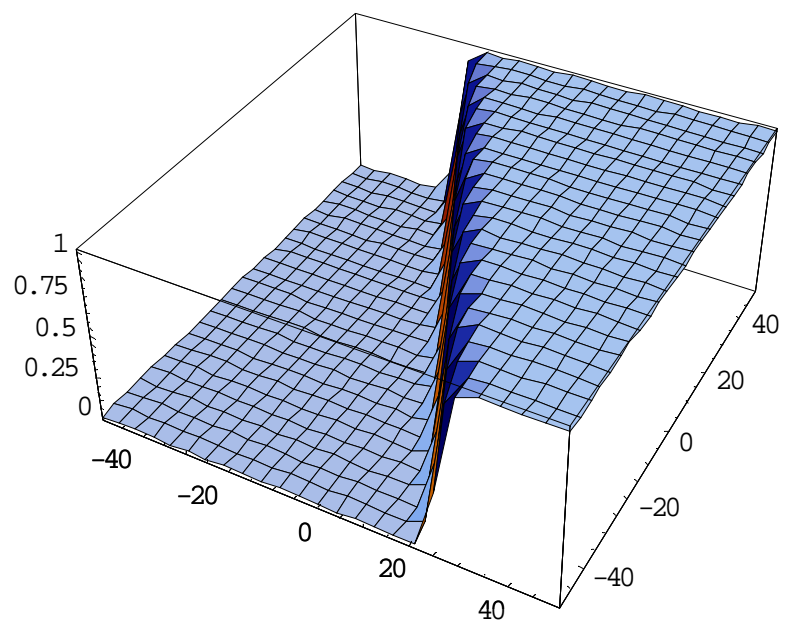

Figure 5 - Solution $u$ is shown at $b_{0}=2$ and $n=1$.

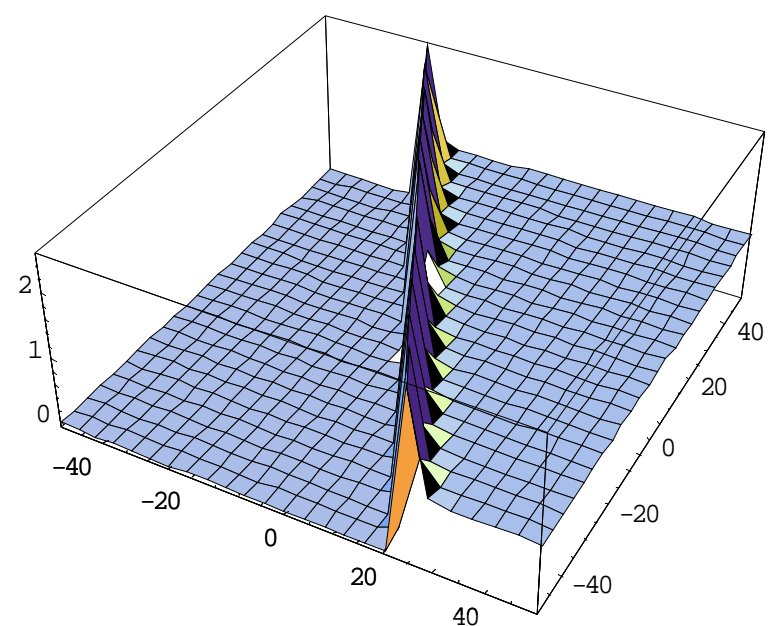

Figure 6 - Solution $u$ is shown at $b_{0}=-2$ and $n=1$.

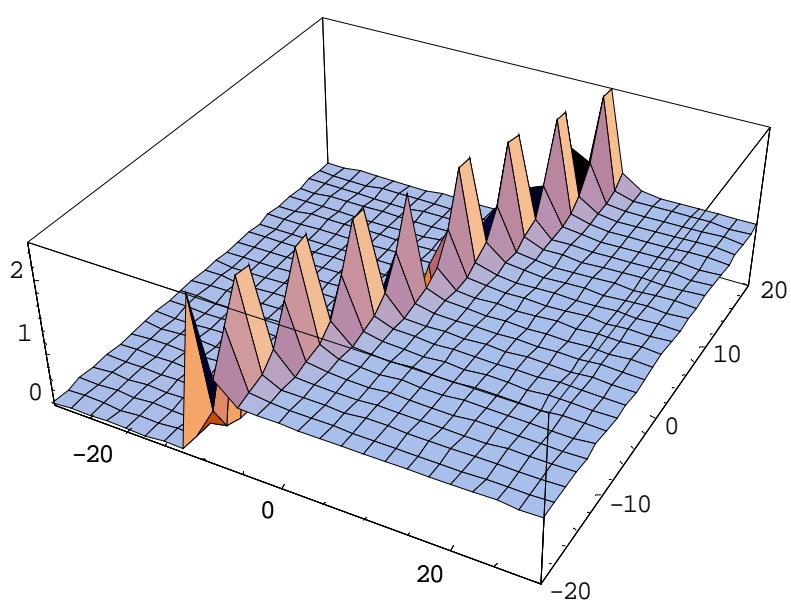

Figure 7 - Solution $u$ is shown at $b_{0}=-2$ and $n=1$. 


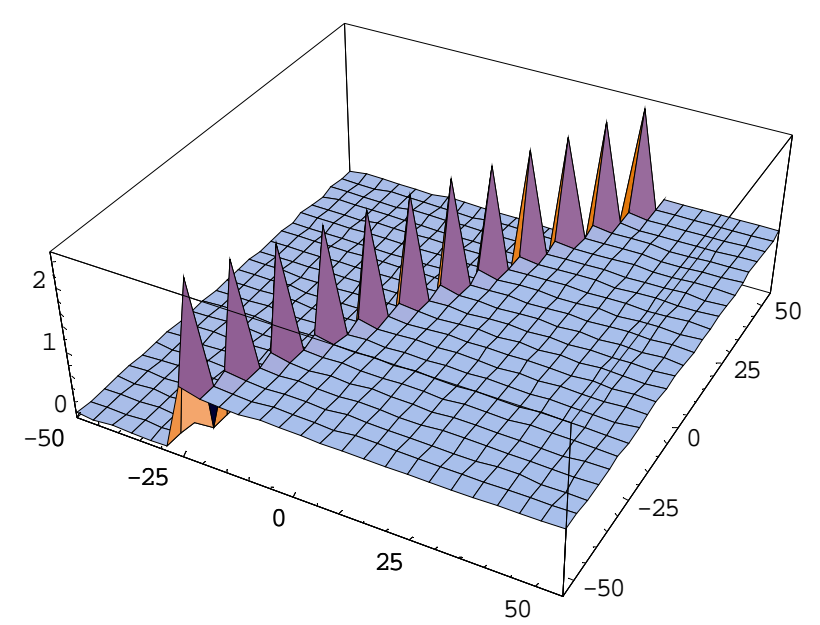

Figure 8 - Solution $u$ is shown at $b_{0}=-2$ and $n=2$.

\section{CONCLUDING REMARKS}

In this study, we applied Exp-function method for obtaining the exact solutions of the two-dimensional Bratu-type equation and the generalized Fisher equation. The results show that this method is a powerful and effective mathematical tool for solving nonlinear evolution equations in science and engineering.

\section{REFERENCES}

1. W. Malfliet, Solitary wave solutions of nonlinear wave equations, American Journal of Physics 60, 650-654, 1992.

2. S. A. El-Wakil and M.A. Abdou, New exact travelling wave solutions using modified extended tanh-function method, Chaos, Solitons and Fractals 31, 840-852, 2007.

3. A. M. Wazwaz, The sine-cosine method for obtaining solutions with compact and noncompact structures, Applied Mathematics and Computation 159, 559-576, 2004.

4. E. Fan and H. Zhand, A note on the homogenous balance method, Physics Letters A 246, 403-406, 1998.

5. C. Q. Dai and J. F. Zhang, Jacobian elliptic function method for nonlinear differential-difference equation, Chaos Solitons and Fractals 27, 1042-1047, 2006.

6. M. A. Abdou, The extended F-expansion method and its applications for a class of nonlinear evolution equations, Chaos, Solitons and Fractals 31, 95-104, 2007.

7. J. H. He, Application of homotopy perturbation method to nonlinear wave equations, Chaos, Solitons and Fractals 26, 695-700, 2005.

8. J. H. He and X. H. Wu, Construction of solitary solution and compaction-like solution solution by variational iteration method, Chaos Solitons and Fractals 29, 108$113,2006$.

9. R. Hirota, Exact solution of the $\mathrm{KdV}$ equation for multiple collisions of solitons, Physical Review Letters 27, 1192-1194, 1971.

10. M. Y. Tang, R. Q. Wang, and Z. J. Jing, Solitary waves and their bifurcations of $\mathrm{KdV}$ like equation with higher order nonlinearity, Science in China Series A 45, 1255 1267, 2002. 
11. J. H. He and X. H. Wu, Exp-function method for nonlinear wave equations. Chaos Solitons and Fractals 30, 700-708, 2006.

12. X. W. Zhou, Y. X. Wen, and J. H. He, Exp-function method to solve the nonlinear dispersive $\mathrm{K}(\mathrm{m}, \mathrm{n})$ equations. International Journal of Nonlinear Science and Numerical Simulation 9, 301-306, 2008.

13. E. Misirli and Y. Gurefe, The Exp-function method to solve the generalized Burgers-Fisher equation. Nonlinear Science Letters A: Mathematics, Physics and Mechanics 1, 323-328, 2010.

14. A. Bekir, Application of the Exp-function method for nonlinear differentialdifference equations, Applied Mathematics and Computation 215, 4049-4053, 2010.

15. E. Misirli and Y. Gurefe, Exact solutions of the Drinfel'd-Sokolov-Wilson equation using the Exp-function method, Applied Mathematics and Computation 216, 2623 2627, 2010.

16. X. H. Wu and J. H. He, Solitary solutions, periodic solutions and compacton-like solutions using the Exp-function method, Computers and Mathematics with Applications 54, 966-986, 2007.

17. A. Ebaid, Exact solitary wave solutions for some nonlinear evolution equations via Exp-function method, Physics Letters A 365, 213-219, 2007.

18. Z. D. Dai, C. J. Wang, S. Q. Lin, D. L. Li, G. Mu, The Three-wave method for nonlinear evolution equations, Nonlinear Science Letters A: Mathematics, Physics and Mechanics 1, 77-82, 2010.

19. S. Zhang, Exp-function method: solitary, periodic and rational wave solutions of nonlinear evolution equations, Nonlinear Science Letters A: Mathematics, Physics and Mechanics 1, 143-146, 2010.

20. Y. Gurefe and E. Misirli, Exp-function method for solving nonlinear evolution equations with higher order nonlinearity, Computers and Mathematics with Applications doi:10.1016/j.camwa.2010.08.060, 2010.

21. J. P. Boyd, An analytical and numerical study of the two-dimensional Bratu equation, Journal of Scientific Computing 1, 183-206, 1986.

22. H. C. Rosu and O. Cornejo, Supersymmetric pairing of kinks for polynomial nonlinearities, Physical Review E 71, 046607, 2005.

23. H. A Abdusalam and E. S. Fahmy, Exact solution for the generalized Telegraph Fisher equation, Chaos Solitons and Fractals 41, 1550-1556, 2009.

24. R. Aris, The Mathematical Theory of Diffussion and Reaction in Permeable Catalyst, Oxford, 1989.

25. A. M. Wazwaz, The tanh-coth method for solitons and kink solutions for nonlinear parabolic equations, Applied Mathematics and Computation 188, 1467-1475, 2007. 